\title{
Prevalence of Psychiatric Comorbidity among Suicide Attempters
}

\author{
Bhuvana Chandrashekarappa Revappala', Sridhar Mallanaik², Vibha Konthanur Vijayakumar³, \\ Suresh Krishnappa Kudumallige ${ }^{4}$, Sarala Nedige Eshwarappa ${ }^{5}$
}

1, 2, 3, 4,5 Department of Psychiatry, Shimoga Institute of Medical Sciences, Shimoga, Karnataka, India.

\section{ABSTRACT}

\section{BACKGROUND}

Psychiatric illness is the most common cause of suicidal attempt which increases the risk further if left untreated. Suicide attempt is one of the forms of suicidal behaviour, which includes suicide ideas, suicide plans and suicide attempts, as these increase the risk further. The purpose of this study was to assess the prevalence and psychiatric comorbidity among suicide attempters.

\section{METHODS}

In this cross-sectional study, samples were collected from patients visited to outpatient department (OPD)/in-patient department (IPD) at a tertiary care center. A total of 50 sample size of both sex with age group of 18 - 65 years are considered. Severity of suicide attempts was assessed by using suicide risks form scale.

\section{RESULTS}

Depression is found to be the most common cause of suicide attempt among mood disorders followed by anxiety disorders, personality disorders and substance use disorders respectively.

\section{CONCLUSIONS}

Our study proved the presence of psychiatric co-morbidity among suicide attempters. Co-morbidity worsens the suicidal risk further as assessed.

\section{KEY WORDS}

Psychiatric Comorbidity, Suicide Attempts.
Corresponding Author: Dr. Sridhar Mallanaik, \#605, Sangam Doctors Quarters, Near IMA House, SIMS Campus, Sagar Road, Shimoga-577201, Karnataka, India.

E-mail:drsridhar2@gmail.com

DOI: $10.14260 /$ jemds $/ 2021 / 684$

How to Cite This Article:

Revappala BC, Mallanaik S, Vijayakumar $V K$, et al. Prevalence of psychiatric comorbidity among suicide attempters. J Evolution Med Dent Sci 2021;10(38): 3370-3373, DOI:

10.14260/jemds/2021/684

Submission 18-02-2021,

Peer Review 06-09-2021,

Acceptance 13-09-2021,

Published 20-09-2021.

Copyright (C) 2021 Bhuvana Chandrashekarappa Revappala et al. This is an open access article distributed under Creative Commons Attribution License [Attribution 4.0 International (CC BY 4.0)] 


\section{BACKGROUND}

Suicide is the act of an instance of taking one's own life voluntarily and intentionally. ${ }^{1} 17 \%$ of people who end their own life are residents of India, whereas 8,00,000 people die by suicide world-wide having the suicide rate increased from $7.9 \%$ - $10 \%$ / 1,00,000 between 1987 - 2007 which increased further to $16 \%$ per $1,00,000$ population according to World Health Organization (WHO), ${ }^{2,3} \mathrm{An}$ attempt to die by suicide resulting in survival is called suicide attempt. Due to acquiring of increased tolerance for physical pain with decreased fear of death with increased capability to lethality leads to engage in supposedly fear-provoking suicidal behaviour. There is also increased risk of completed suicide which is ten to hundred times higher than the general population. Even though clinician put efforts in monitoring the suicide attempts, $10 \%-15 \%$ have completed the suicide. ${ }^{4,5}$

A study by Beurs et al. states that there is less attention given on prospective association of suicide attempt i.e. association of the suicide attempt to mental disorders. i.e. suicide attempts lead to mental disorder within 6 years of follow up period in the study. ${ }^{6}$ Many researchers have identified multiple risk factors for suicide: Individual level co-morbid psychiatric disorder, previous suicide attempt, social isolation, financial problems, substance use disorders, impulsive tendencies; Relationship level - child abuse and neglect, history of suicide in family, love breakup, loss of loved ones, sexual abuse and violence, negative interfamily interaction; Community level - failure to get health care services, cultural and religious belief such as belief, massive suicide in the community; Societal - stigma associated with mental illness or help seeking.7,8 Presence of two or more mental illness worsens the risk of suicide further. The stages of suicide progress from suicide ideation to plan to attempt. Different stages of attempt are associated with unique psychiatric disorders. Depression is linked to suicide ideation than suicide plan or attempt; and, in contract, the symptoms of anxiety or impulse control difficulty are more linked to plan or attempt among ideates. ${ }^{9}$ There is a gap in the knowledge of which psychiatric disorder is associated with suicidal behaviours, and the manner in which comorbidity contributes to these outcomes, is needed for better understanding. According to the study by Stein et al. the multiple suicide attempters have relatively higher level of anti-social behaviour and also state that suicide groups may differ in terms of psychiatric comorbidity. ${ }^{10}$

The presence of psychiatric illness worsens the risk of suicide and hence the need for study. Our study aimed at prevalence of psychiatric co-morbidity among suicide attempters.

\section{METHODS}

It is a cross-sectional study conducted at Shimoga Institute of Medical Sciences, Shimoga, Karnataka from $1^{\text {st }}$ January 2021 to March 2021. A total of 50 subjects of both sexes whose age group of 18 - 65 years were consider for the study. Size was based on inclusion and exclusion criteria. Samples were collected from the suicide attempters who visited OPD/IPD.
Convenience sampling method was used because participants are selected based on availability and willingness to take part. Psychiatric comorbidity is assessed using International Classification of Diseases (ICD-10 classification system). Suicide risks assessment form was used to assess the suicide risk. It consisted of 15 questionnaires with each having three sub-questions and each score was graded from 0 - 2. Scores were graded as follows: 0 - 10: low risks, $11-20$ : medium risks and 20 - 30: high risks. High risk needs immediate attention and needs admission by psychiatrist immediately. IPDE (identify, predict, decide and execute) is used to assess personality disorders.

\section{Inclusion Criteria}

1. All suicide attempters.

2. First psychiatric consultation.

\section{Exclusion Criteria}

1. Mental retardation.

2. Chronic disabling medical conditions like epilepsy, hypertension and diabetes mellitus.

3. Cases of psychotic disorders.

4. Suicide attempt under the influence of alcohol.

\section{Ethical Clearance of Institution}

Obtained: (SIMS/IEC/611/2021-22)

\section{Statistical Analysis}

Results were tabulated in the form of graph.

\section{RESULTS}

Overall, $92 \%$ of the people were suffering from the comorbid psychiatric illness among suicide attempters. $63 \%$ of the study sample were suffering from mood disorders; out of which, $55 \%$ were suffering from depressive disorders, $2 \%$ with dysthymia, $5 \%$ with recurrent depressive disorders and $1 \%$ with bipolar affective disorders.

\begin{tabular}{|cc|}
\hline Mood Disorders & \\
Depressive disorder & $55 \%$ \\
Dysthymia & $2 \%$ \\
Recurrent depressive disorder & $5 \%$ \\
Bipolar affective disorder & $1 \%$ \\
Total & $63 \%$ \\
\hline Table 1. Prevalence of Psychiatric Co-Morbidity of Mood Disorders \\
among Suicide Attempters
\end{tabular}

\begin{tabular}{|cc|}
\hline & \\
\hline Anxiety Disorders & $5 \%$ \\
Generalised anxiety disorder & $0 \%$ \\
Panic disorder & $3 \%$ \\
Mixed anxiety and depression & $1 \%$ \\
Phobia disorder & $1 \%$ \\
OCD & $2 \%$ \\
Post PTSD & $1 \%$ \\
Somatoform disorder & $\mathbf{1 4} \%$ \\
Total & \\
\hline Table 2. Prevalence of Psychiatric Co-Morbidity of Anxiety Disorders \\
among Suicide Attempters
\end{tabular}

Anxiety disorder is the second most common cause of suicidal attempt $(14 \%)$. Out of which, $5 \%$ were having 
generalised anxiety disorder, panic disorder ( $0 \%)$, mixed anxiety and depression (3\%), phobia disorder (1 \%), obstructive compulsive disorder (OCD) (1\%), post posttraumatic stress disorder (PTSD) (1\%) and somatoform disorder (1\%).

Other disorders include personality disorders which are $10 \%$, substance use disorders $5 \%$ and schizophrenia and psychosis $3 \%$.

\begin{tabular}{|cc|}
\hline Other Disorders & $\mathbf{1 0 \%}$ \\
Personality disorders & $\mathbf{5 \%}$ \\
Substance use disorders & $\mathbf{3 \%}$ \\
\hline Schizophrenia and psychosis \\
\hline Table 3. Prevalence of Psychiatric Co-Morbidity of Other Disorders \\
among Suicide Attempters
\end{tabular}

\section{DISCUSSION}

Our study has proved that there is an association between psychiatric co-morbidity and suicide attempt. The present study repeatedly shows that suicide is most common in mood disorders especially depressive disorders. The time spent during the depressive phase especially with anxiety symptoms is the strongest predictor of suicide attempt. Few patients experienced anxiety and increased depressive symptoms when antidepressant started which leads to suicidality. Based on the above findings, it's better to monitor those who are on antidepressant which are not lethal if taken overdose, providing alert to family members regarding worsening of the symptoms or suicidal ideation. The relationship between anxiety disorder and suicide is the progression from suicide ideation to plan to attempts. Some study states that there might be relation between anxiety disorder and suicide behaviours, while other studies state that anxiety disorder on their own can't be effective in triggering suicidal behaviour. Anxiety disorders are often cooccurring with depression and this may be masking a risk of suicidal behaviour specifically related to anxiety. Among anxiety disorders, OCD and PTSD are linked to high rate of suicide. ${ }^{11,12}$

Badrinarayana noted $65.2 \%$ cases having psychiatric diagnosis, with depression in $48 \%$ being most frequent. ${ }^{12,13}$

Morgan et al. assessed 368 patients who presented following some form of deliberate self-harm. $90 \%$ were considered to have some form of mental illness, of which neurotic depression (52\%) being most common. ${ }^{14}$

Roy and Chir studied 243 patients with a family history of suicide, of whom $48.6 \%$ had attempted suicide. Of these cases, $56.4 \%$ had a depressive disorder and $34.6 \%$ had recurrent depressive disorder. ${ }^{15}$

Suominen et al. reported that $98 \%$ of suicide attempters had one or more psychiatric diagnoses and in only one case no diagnosis could be made. Depressive disorders were the most common disorders. Major depression was diagnosed in $38 \%$ of cases and depressive syndrome was diagnosed in 75 $\%$ of cases. ${ }^{16}$

In a case-control study by Beautrais et al. $90.1 \%$ of those who made serious suicide attempt had mental disorder at the time of the attempt. Of these, $76.8 \%$ had mood disorders (61.9\% major depression), $26.8 \%$ had anxiety disorders (phobic disorder $15.9 \%$, panic disorder $10.9 \%$ ). ${ }^{17}$
In a case-control study by Johnson et al. suicide attempters were more likely to have affective disorders than the controls. Affective disorder was present in $72.6 \%$ of attempters and $41.4 \%$ of controls, with major depression (61.3\% v. $35.7 \%$ ) being most frequent. ${ }^{18}$

Haw et al. reported that $92 \%$ of the sample had at least one psychiatric disorder at the time of the index attempt. 72 $\%$ had affective disorder, with $70.7 \%$ having depressive episodes. However, depressive spectrum disorders were present in $78 \%{ }^{19}$

A study by Wunderlich et al. of all suicide attempters, 91 $\%$ had at least one mental disorder, $79 \%$ were co-morbid or multimorbid respectively and $45 \%$ had four or more diagnoses (only $5 \%$ in the total sample reached such high levels of co-morbidity). Suicide attempters with more than three diagnoses were 18 times more likely $(\mathrm{OR}=18.4)$ to attempt suicide than subjects with no diagnosis. ${ }^{20}$

There is a need of more focused care during the first two years of suicide attempt. Because the chances of co-existent, psychiatric morbidity in people who have previously attempted suicide is related to risk of eventual death from suicide.

Person with somatoform disorder or medically unexplained physical pain leads to suicidal attempt to get relief from distress associated with physical concerns or hopelessness derived from the belief that physical pain or concern would not improve, which could contribute to attempted suicide without strong desire to die.

Study of Demore et al. expressed the relatively high impact of suicide attempts among substance misuse or dependence. The hazard ratio between males to female is 2 : 1 with drug misuse or dependence who had attempted suicide. Psychiatric and psychosocial interventions at emergency medical care facilities is required and follow-up care for suicide attempters is considered as an important component for suicide prevention.

\section{CONCLUSIONS}

Knowledge of which disorders that are uniquely predictive of suicidal behaviours, and the manner in which comorbidity contributes to these outcomes, is needed to better understand the mechanisms through which mental disorders lead to suicidal behaviour. In the absence of effective measures against suicide attempters, it has been difficult to reverse an increasing suicide trend. Case management by multi-sector collaboration is required.

\section{Limitations of the Study}

1. Our study doesn't demonstrate socio-demographic factors.

2. Our study result is in-compactible to other study findings due to stigma associated with suicide that are underreported.

3. It fails to prove suicide risks further due to crosssectional study.

Data sharing statement provided by the authors is available with the full text of this article at jemds.com.

Financial or other competing interests: None. 
Disclosure forms provided by the authors are available with the full text of this article at jemds.com.

\section{REFERENCES}

[1] Janet W, Therese SR, Joseph C, et al. Military veteran mortality following a survived suicide attempt BMC Public Health 2011;11:374.

[2] Hirayasu Y, Kawanishi C, Yonemoto N, et al. A randomized controlled multicenter trial of post-suicide attempt case management for the prevention of further attempts in Japan (ACTION-J). BMC Public Health 2009;9:364.

[3] Nock MK, Borges G, Bromet EJ, et al. Cross-national prevalence and risk factors for suicidal ideation, plans and attempts in the WHO World Mental Health Surveys. Br J Psychiatry 2008;192(2):98-105.

[4] Borges G, Angst J, Nock MK, et al. Risk factors for the incidence and persistence of suicide-related outcomes: a 10-year follow-up study using the National Comorbidity Surveys. J Affect Disord 2008;105(1-3):25-33.

[5] Tidemalm D, Långström $\mathrm{N}$, Lichtenstein $\mathrm{P}$, et al. Risk of suicide after suicide attempt according to coexisting psychiatric disorder: Swedish cohort study with long term follow-up. BMJ 2008;337:a2205.

[6] Johannessen HA, Dieserud G, Leo DD, et al. Chain of care for patients who have attempted suicide: a follow-up study from Bærum, Norway. BMC Public Health 2011;11:81.

[7] Fernandez de la Cruz L, Rydell M, Runeson B, et al. Suicide in obsessive-compulsive disorder: a populationbased study of 36[thinsp]788 Swedish patients. Mol Psychiatry 2016;22(11):1626-32.

[8] Wilcox HC, Storr CL, Breslau N. Posttraumatic stress disorder and suicide attempts in a community sample of urban American young adults. Arch Gen Psychiatry 2009;66(3):305-11.
[9] Panagioti M, Gooding PA, Tarrier N. A meta-analysis of the association between posttraumatic stress disorder and suicidality: the role of comorbid depression. Compr Psychiatry 2012;53(7):915-30.

[10] Hawton K, Sutton L, Haw C, et al. Schizophrenia and suicide: systematic review of risk factors. Br J Psychiatry 2005;187:9-20.

[11] Hawton K, Sutton L, Haw C, et al. Suicide and attempted suicide in bipolar disorder: a systematic review of risk factors. J Clin Psychiatry 2005;66(6):693-704.

[12] De Moore GM, Robertson AR. Suicide in the 18 years after deliberate self-harm: a prospective study. $\mathrm{Br} \mathrm{J}$ Psychiatry 1996;169(4):489-94.

[13] Badrinarayana A. Suicidal attempt in Gulbarga. Indian J Psychiatry 1977;19(4):69-70.

[14] Morgan HG, Burns-Cox CJ, Pocock H, et al. Deliberate selfharm: clinical and socio-economic characteristics of 368 patients. Br J Psychiatr 1975;127:564-74.

[15] Roy A. Family history of suicide. Arch Gen Psychiatry 1983;40(9):971-4.

[16] Suominen K, Henriksson M, Suokas J, et al. Mental disorders and comorbidity in attempted suicide. Acta Psychiatr Scand 1996;94(4):234-40.

[17] Beautrais AL, Joyce PR, Mulder RT, et al. Prevalence and comorbidity of mental disorders in persons making serious suicide attempts: a case-control study. Am J Psychiatry 1996;153(8):1009-14.

[18] Johnson BA, Brent DA, Bridge J, et al. The familial aggregation of adolescent suicide attempts. Acta Psychiatr Scand 1998;97(1):18-24.

[19] Haw C, Hawton K, Houston K, et al. Psychiatric and personality disorders in deliberate self-harm patients. $\mathrm{Br}$ J Psychiatry 2001;178(1):48-54.

[20] Wunderlich U, Bronisch T, Wittchen HU. Comorbidity patterns in adolescents and young adults with suicide attempts. Eur Arch of Psychiatry and Clin Neurosci 1998;248(2):87-95. 